\title{
Structural Analysis of a Dragonfly Wing
}

\author{
S.R. Jongerius • D. Lentink
}

Received: 30 August 2009/Accepted: 8 September 2010/Published online: 26 October 2010

(C) The Author(s) 2010. This article is published with open access at Springerlink.com

\begin{abstract}
Dragonfly wings are highly corrugated, which increases the stiffness and strength of the wing significantly, and results in a lightweight structure with good aerodynamic performance. How insect wings carry aerodynamic and inertial loads, and how the resonant frequency of the flapping wings is tuned for carrying these loads, is however not fully understood. To study this we made a three-dimensional scan of a dragonfly (Sympetrum vulgatum) fore- and hindwing with a micro-CT scanner. The scans contain the complete venation pattern including thickness variations throughout both wings. We subsequently approximated the forewing architecture with an efficient three-dimensional beam and shell model. We then determined the wing's natural vibration modes and the wing deformation resulting from analytical estimates of 8 load cases containing aerodynamic and inertial loads (using the finite element solver Abaqus). Based on our computations we find that the inertial loads are 1.5 to 3 times higher than aerodynamic pressure loads. We further find that wing deformation is smaller during the downstroke than during the upstroke, due to structural asymmetry. The natural vibration mode analysis revealed that the structural natural frequency of a dragonfly wing in vacuum is $154 \mathrm{~Hz}$, which is approximately 4.8 times higher than the natural flapping frequency of dragonflies in hovering flight $(32.3 \mathrm{~Hz})$. This insight in the structural properties of dragonfly wings could
\end{abstract}

S.R. Jongerius $\cdot$ D. Lentink $(\bowtie)$

Faculty of Aerospace Engineering,

Delft University of Technology,

2600 GB Delft, The Netherlands

e-mail: david.lentink@wur.nl

S.R. Jongerius $\cdot$ D. Lentink

Experimental Zoology Group, Wageningen University,

6709 PG Wageningen, The Netherlands inspire the design of more effective wings for insectsized flapping micro air vehicles: The passive shape of aeroelastically tailored wings inspired by dragonflies can in principle be designed more precisely compared to sail like wings - which can make the dragonfly-like wings more aerodynamically effective.

Keywords Dragonfly - Insect wing - Micro-CT scan · FEM . Structural analysis $\cdot$ Inertia $\cdot$ Aerodynamics

\section{Introduction}

Recently designed flapping micro air vehicles have been developed inspired by insect flight [1-3]. These flapping MAVs generate both lift and thrust by flapping their sail-like wing structures that deform primarily due to aerodynamic loading [3], Fig. 1. Whereas flapping MAVs typically employ slack wings, the wings of most insects are very stiff compared to the sail-like wing structures of MAV wings (e.g. [4]). How insect wings function structurally under dynamic wing loading is not yet fully understood, although existing studies have generated novel insight in how the venation pattern of insects is critical for their load baring capacity and aeroelastic function. Counter-intuitively the corrugated wings of insects provide good aerodynamic performance at low Reynolds numbers [5-9], while providing good structural strength and stiffness [6]. Numerical analyses of insect wings have been performed by Smith [10], Kesel et al. [11], Herbert et al. [12], Combes and Daniel [13-15] and Wootton et al. [16] to better understand the structural function of insect wings under different loading regimes. Smith [10] performed a modal analysis of a quasi-two-dimensional finite element model (FEM) of the fore- and hindwing of a moth, to correlate the 


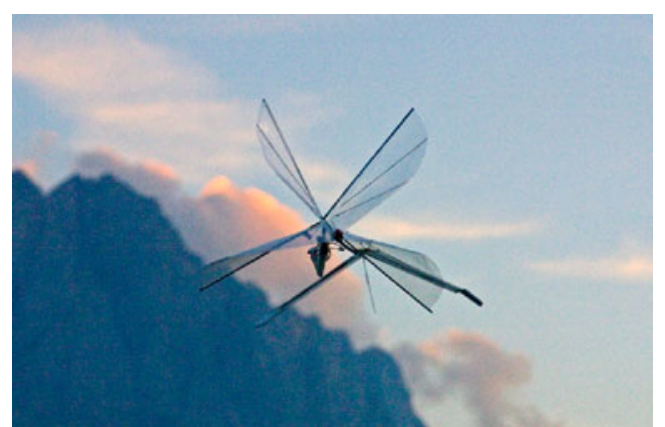

Fig. 1 DelFly, a flapping wing micro aerial vehicle inspired by insect flight with slack sail-like wings [3]

distribution of mass and stiffness. Kesel et al. [11] increased model fidelity to study structural stabilization of dragonfly wings by vein corrugation. Herbert et al. [12] numerically explored the mechanisms that lead to the umbrella effect, a mechanism of camber generation in the hindwing fans of orthopteroid and dictyopteroid insects, as described by Wootton [17]. They built cambered FEMs of the hindwing of a grasshopper (Schistocerca gregaria). Combes and Daniel [15] created a quasi- two-dimensional FEM to explore how damping affects a Manduca sexta forewing. Wootton et al. [16] performed a modal and impact analysis on a slightly cambered simplified model of a Manduca sexta wing. Their results indicate that the natural vibration frequency of a Manduca wing is equal to the flapping frequency of Manduca in flight. They also addressed several challenges previous studies encountered in accurately describing wing morphology.

Here we focus on the three-dimensional structural function of dragonfly wings (Fig. 2), because their venation pattern has remained surprisingly similar during their evolution, suggesting this conserved feature plays an important role in flight. The Protodonata, the ancestors of the dragonflies (Odonata), were among the earliest fliers in the Carboniferous.

Approximately 350 million year old fossils of Meganeuridae tell us that they flew with wingspans of up to $75 \mathrm{~cm}$. Their wings are similar enough to modern shapes to suggest comparable flight capabilities, although perhaps with less refinement [18, 19]. Modern dragonfly wings have similar wing architecture, but are much smaller in size. The relatively constant wing architecture across dragonfly evolution suggests that the wing architecture of dragonflies functions well over a large range of wingspans. Furthermore, dragonflies are among the more agile flyers. They hunt in flight, are highly manoeuvrable and even mate mid-air. Dragonflies often cope with accelerations of up to $4 \mathrm{~g}$ in a straight line and $9 \mathrm{~g}$ in steep turns as documented in high speed video recordings [20]. This shows that dragon- fly wings most likely have evolved into effective wings that function well under diverse high-performance flight conditions. Understanding how these wings function structurally could, therefore, inspire the design of more effective wings for insect-sized micro air vehicles.

In our structural analysis of dragonfly wings we digitized a dragonfly fore- and hindwing. The geometry of the forewing was captured accurately enough to be modeled well with an array of linked beams and shells to obtain an efficient Finite Element Model. We use this FEM to reveal deformations, and natural vibration characteristics of a dragonfly forewing, to obtain better insight in the functional morphology of dragonfly wings.

\section{Materials and Methods}

We chose to study dragonfly (Sympetrum vulgatum) wings. The fore- and hindwing were digitized using the micro computed tomography (micro-CT) scanning method. The forewing geometry is subsequently converted into a FEM for the Abaqus (6.5-1, Dassault Systèmes) solver. For structural analysis of the wing we gathered wing cuticle properties and wing kinematics from literature. Thereupon we made a simplified analytical model of the aerodynamic and inertial wing loads during hovering flight. Combined, we obtain a three-dimensional structure and load model of a dragonfly wing.

\section{Insect Collection and Treatment}

We caught a dragonfly (Sympetrum vulgatum) near a pond in the mid-eastern part of The Netherlands. To preserve the dragonfly we stored it in a one to one solution of water and alcohol, directly after we gassed the dragonfly with ethyl-acetate.

\section{Micro CT Scan of Dragonfly Wing}

After exploring several digitizing methods such as stereo photography, 3D laser scanning [21] and wing sectioning techniques we selected the micro computed tomography (micro-CT) technique. A micro-CT scanner creates a 3D stack of cross-sections throughout the wing based on X-ray imaging. SkyScan at Anvers (Belgium) kindly sponsored two days of scanning time on their high-resolution SkyScan 1172 micro-CT scanner. This proved to be enough time for scanning one $(n=1)$ fore- (single wing span $28,8 \mathrm{~mm}$ ) and hindwing (single wing span $26,8 \mathrm{~mm}$ ) after several initial trials. Before scanning, we dried the wing for twelve hours at room temperature. Drying deforms the wing but is necessary to reduce noise and scatter. Both fore- and hindwing of Sympetrum vulgatum were scanned with a 
Fig. 2 Photograph (a) of a dragonfly Sympetrum vulgatum forewing with inset images $(b \mathrm{t} / \mathrm{m} g)$ of the wings detailed structure captured by scanning electron microscopy (positions are approximated). Leading and trailing edge protuberances are believed to produce an increase in lift [43]. (b) Protuberances on leading edge. (c) The nodus of the wing. (d) The wing tip. (e) Vein crossing near the wing root. (f) Trailing edge with protuberances. (g) Wing surface near the wing tip
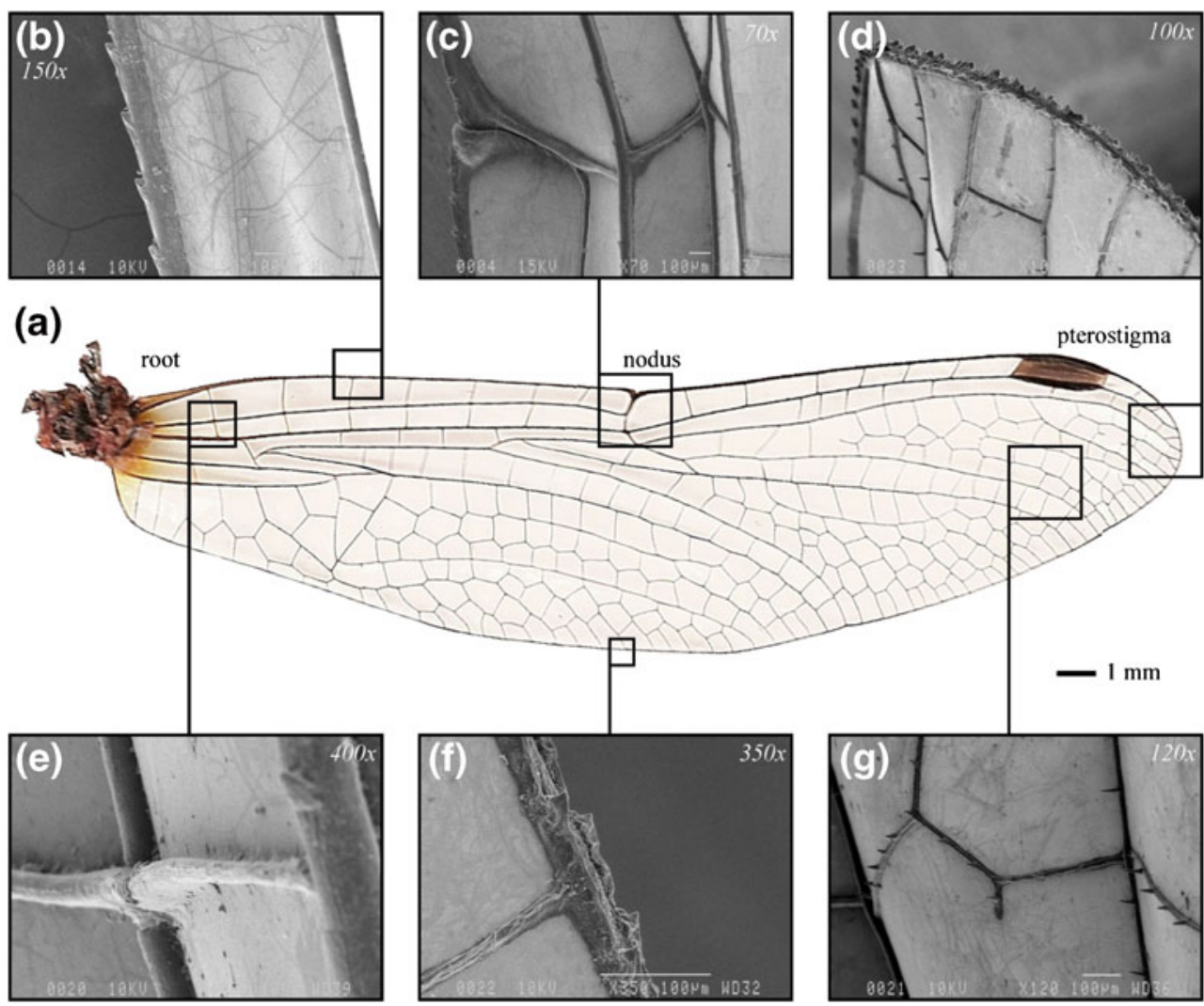

resolution of $7.2 \mu \mathrm{m}$. This means that every pixel in each of the resulting cross-sectional images has a dimension of $7.2 \times 7.2 \mu \mathrm{m}$. Also the distance between two successive images in the stack is $7.2 \mu \mathrm{m}$. The scans resulted in a total of 3,997 cross-sectional images for the forewing and 3,718 images for the hindwing. The scan provides us with three dimensional information of the dragonfly wing, including vein corrugation and thickness distribution. We wrote a dedicated Matlab program (Matlab 7.0; The Mathworks) to digitally smooth the cross-sections and subsequently reconstruct the wings (Figs. 3 and 4). The gray-values in Figs. 3 and 4 represent wing thickness. Dark indicates thick areas whereas light areas are thin. Although we used a state of the art micro-CT scanner, dragonfly wings still proved to be challenging thin. Especially for the hindwing in Fig. 4; 56\% of its membrane area has a thickness less than the resolution of the scan. Fortunately, roughly $96 \%$ of the area of the forewing proved thick enough for a threedimensional scan. We therefore focus our study on the forewing and assume that the area with a thickness smaller than the scan resolution (approximately 4\%) has a thickness of $3.6 \mu \mathrm{m}$; half the scan resolution.

\section{The Finite Element Model}

We wrote dedicated Matlab software to convert the digitized three-dimensional geometry into a suitable finite element model to analyze the mechanical function of the wing under representative loading conditions. Finite element models can be made out of volumetric elements or structural elements stretched between nodes. We chose to use structural elements because this results in effective data handling and an easy adaptable model structure. Beam elements (Abaqus: B31 elements) are used to represent wing veins, the membranes between the veins were represented by thin-shell elements having small strain (Abaqus: STRI3 elements). The elements are stretched between nodes assigned at the positions of crossing veins. Based on the micro-CT scan, nodes and elements were automatically assigned by custom 3D Matlab scripts. In total, the forewing was modeled with the use of 543 nodes, 823 beams and 998 shell elements. We could not further refine the FEM model due to the complexity involved to program this without sacrificing the geometric accuracy. To check if the number of elements in our FEM model was appropriate for numerical convergence we performed a literature survey. Combes and Daniel [13-15] found an asymptotic solution for their FEM model of a Manduca sexta wing for 865 elements and up (FEM solver MSC Marc/Mentat 2001). Considering the similarity in wing dimensions and number of elements and the quality of the solution we obtained, we are confident we performed an accurate FEM analysis. 
Fig. 3 (a) Digital reconstruction of the forewing of Sympetrum vulgatum. Dark areas are thick, light areas are thin (linear scale from dark to light). (b-e) Crosssectional micro-CT image near the wing root showing the highly corrugated wing architecture build up by the wings veins and membrane. Cross sections more distant of the root (c-e) show less corrugation and the cross section near the wing tip (e) reveils the hollow pterostigma

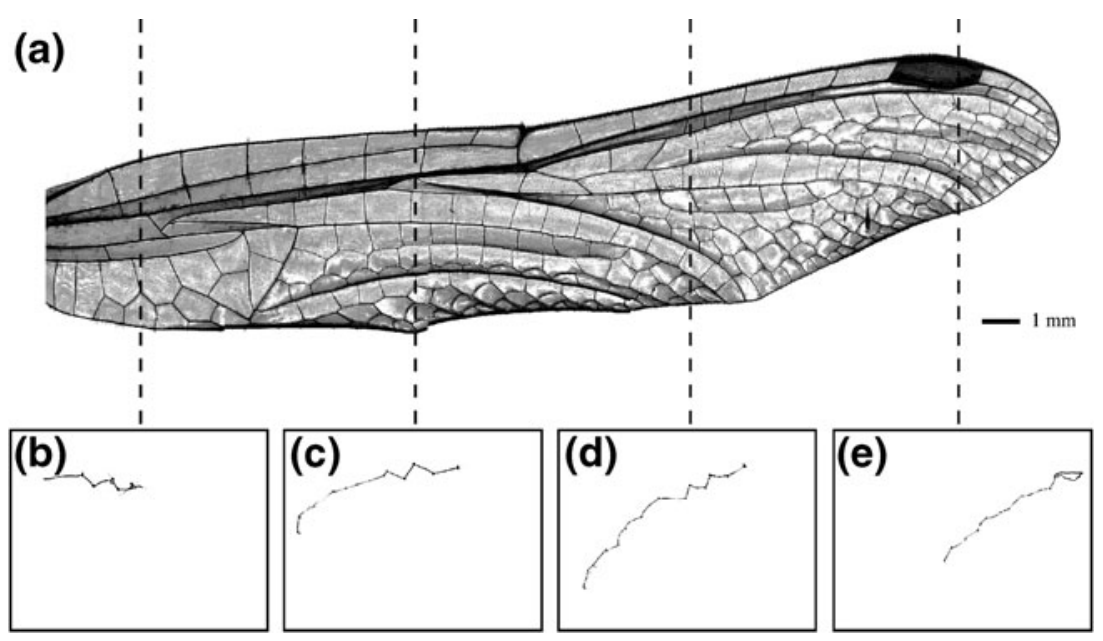

The thickness of each element was determined based on the micro-CT image stack by a custom Matlab script. The beam element thicknesses are averages of the thicknesses around the nodes of the beams (in a $10 \times 10$ pixel area). The cross-sections of the beams were defined as hollow pipes with a wall thickness of $3 / 4$ th of the beam radius. We measured this as an average in ten cross-sectional micro-CT images evenly distributed over the wing length. To calculate the average thickness of each membrane we took $10 \%$ of each membrane area at its centre. The thickness distribution is manually checked for anomalies. We then adapted values of 17 beam elements and 4 shell elements to the average of surrounding thickness values to correct identified anomalies. The resulting model provides us with an accurate shell and beam thickness distribution of the wing. The wing model is fixed by restricting all degrees of freedom of four nodes at the root. The micro-CT scan shows much scatter around this area because of remaining insect body parts. We therefore manually model the wing root approximating the root shown in Fig. 2. Loads are modeled as pressure loads using a quasi steady model, neglecting unsteady aerodynamic effects. The aerodynamic load acting on each shell element is modeled as a uniform pressure load calculated using an analytic pressure distribution model. Inertial loads were modeled as line pressure loads on beams and uniform pressure loads on shell elements. To perform the FEM analysis we developed custom Matlab software that generates an input file for the Abaqus solver. This text file contains information regarding the geometry, material properties, loads and type of
Fig. 4 (a) Digital reconstruction of the hindwing of Sympetrum vulgatum. Dark areas are thick, light areas are thin (linear scale from dark to light). (b-e) Crosssectional micro-CT image near the wing root showing the highly corrugated wing architecture build up by the wings veins and membrane. Cross sections more distant of the root (c-e) show less corrugation and the cross section near the wing tip (e) reveils the hollow pterostigma. The main difference between this figure and 3 are the much thinner veins and membrane

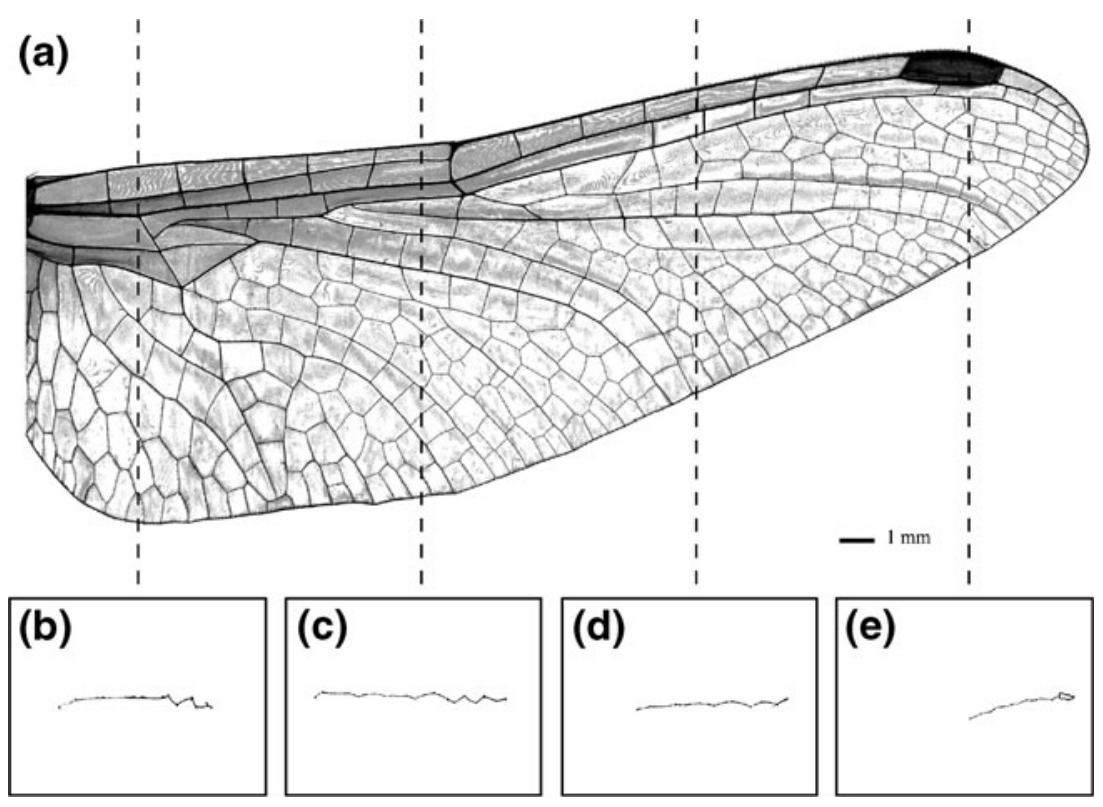


analysis. Abaqus statically analyzed the wing deformations and stresses. Also free vibration analyses were performed to extract the wing's natural vibration modes. The latter is done by a perturbation step in which the natural vibration frequency is calculated using the Lanczos method [22]. We performed an analysis of the natural vibration modes of a two- and three-dimensional geometrical wing model (in the 2-D model we made the $\mathrm{z}$-coordinate identical for all nodes; note that doing this the vein and membrane models remained 3D). Comparing the results of non-linear and linear simulations gave similar results, because the deformations are relatively small (less than $5.5 \%$ of wing length). Therefore we decided to perform linear analysis, neglect nonlinear geometric effects, to reduce computational time.

\section{Dragonfly Wing Material Properties}

For the Finite Element Analysis we needed the material properties of our dragonfly (Sympetrum vulgatum) wing. Therefore we collected averaged data from literature. The material density of wing cuticle $\rho_{\mathrm{c}}$ is taken as $1,200 \mathrm{~kg} / \mathrm{m} 3$ (measured in biological materials by Wainwright et al. [23], and confirmed by Vincent and Wegst [24]) and we used a Poisson's ratio $v$ of 0.49 [23]. Combes and Daniel [13-15] also used a Poisson's ratio of 0.49 and tested the effects of using a Poisson's ratio of 0.3 and found the effect to be negligible. In our analysis we took the material stiffness of the wing (Young's modulus, E) constant throughout the wing for both the veins and membranes, similar to Smith [10] and Kesel et al. [11]. We further took an average vein stiffness of $6 \mathrm{GPa}$ [12], and for the membrane stiffness we took a value of $3.75 \mathrm{GPa}$ [24]. Some studies revealed that the Young's modulus can vary widely within a wing [25] and that some proteins, such as resilin, can alter local properties [26, 27]. However, measuring spatial material property variations accurately in concert with the $3 \mathrm{D}$ wing structure is still out of scope of present technology.

To calculate wing loads during hovering flight, insect properties like body mass, wing mass and wing kinematics are needed. Because we immediately preserved our sample from drying we used the average Sympetrum vulgatum body mass as $225 \mathrm{mg}$ as measured for three samples by Rüppell [28]. The wing mass was calculated by multiplying the wing volume, as measured in the micro-CT image stack, by the cuticle density. The total wing mass appeared to be $3.4 \mathrm{mg}$, which is $1.5 \%$ of its body mass. This value is in the usual range of wing to body mass ratios; usually between $0.5-5 \%$ of body mass; measured by Ellington [29]. The mass of haemolymph in the hollow veins was taken into account. We took equal density for cuticle and haemolymph and assume this error to be negligible. Possible flow of heamolymph within the veins is also neglected.

\section{Dragonfly Wing Kinematics}

In one flap cycle, the wing revolves around the hinge back and forth at roughly constant angle of attack, while rotating along the spanwise axis during stroke reversal at the beginning and end of a stroke; pronation and supination. When rotating from down- to upstroke, the morphological underside of the wing rotates to face upwards and this is called supination. From up- to downstroke the morphological underside of the wing rotates to face downwards again and this is called pronation. We modeled the flapcycle as a sinusoidal motion:

$$
\varphi(t)=\Phi \cdot \sin \left(\omega_{\text {flap }} t\right)
$$

Here $\varphi(t)$ is the stroke angle of the wing, which depends on time $t$. The flapping frequency $f_{\text {flap }}$ is taken as $32.3 \mathrm{~Hz}$, which yields angular frequency $\omega_{\text {flap }}=2 \pi f=203 \mathrm{rad} / \mathrm{s}$, measured by Rüppell [28]. The flapping amplitude $\Phi$ is taken to be $60^{\circ}$, which is a representative average value for Odonata flapping amplitudes $[28,30]$. A full stroke covers twice the (mathematical) wing stroke amplitude; from peakto-peak the wing revolves over an angle of $120^{\circ}$. Usually, the wing follows a figure eight-like path during the stroke cycle. This occurs in a plane with an inclination angle $(\beta)$ with the horizontal [30]. For simplicity we assume that the wings flap in a horizontal stroke plane during hovering insect flight as described by Ellington [18] (Fig. 5). The angle of attack of the wing during flapping is assumed to be $30^{\circ}$ on average for both the up- and downstroke (Fig. 5), which we estimated based on values found for several insects in literature [31, 32, 38].

\section{Dragonfly Forewing Loading}

During flapping, the wing experiences both inertial and aerodynamic loads that need to be carried by the wing structure. Previous studies [13, 15, 29, 33, 34] suggested that the inertial loads are higher than the aerodynamic loads, from 2 [31] up to 10 times [34]. If such a load ratio also holds for dragonfly wings is unknown. To calculate the wing loads we made an analytical model of both the aerodynamic load needed to support the weight of the animal, and the inertial loads that result from the flapping motion of the wing that can support the weight.

\section{Aerodynamic load model}

We focused on the lift forces the wing needs to bear to oppose body weight, in this force calculation we neglected the loading of the wing due to drag force. Lift in flapping flight is generated by three distinct mechanisms: delayed stall, 


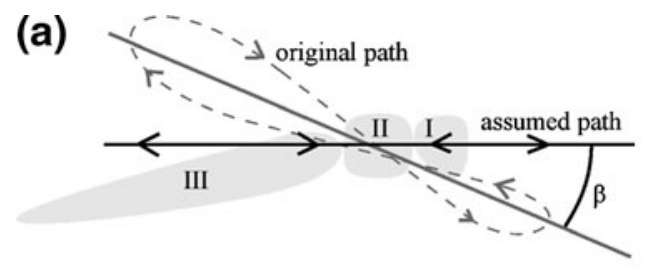

(b)

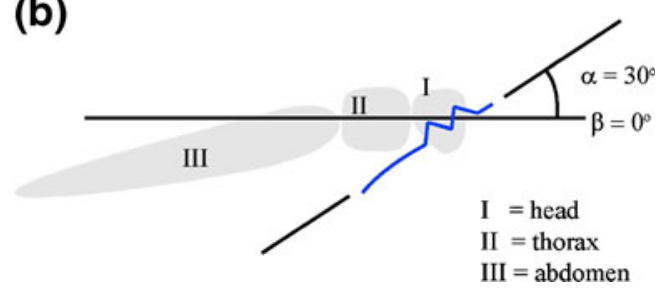

Fig. 5 Side view insect body. (a) Stroke plane is assumed to the horizontal. (b) Angle of attack is assumed to be $30^{\circ}$

rotational circulation and wake capture. Delayed stall generates a leading edge vortex (LEV) during the swing phases of the stroke [35-38]. Rotational circulation and wake capture occur primarily during stroke reversal, while the LEV is prominent mid stroke. Because the total lift generated during the swing phase of the wing is typically considerably higher than the lift generated during stroke reversal [38], we focused on lift generated by delayed stall; the LEV. Another aerodynamic load component is known as 'added mass' or 'virtual mass' [39]. The added mass varies from 0.3 up to 1.2 times the wing mass [33, 40, 41]. Added mass is difficult to model accurately and smaller than lift force, therefore we neglect it in our basic force model. Finally we ignore drag force altogether, because an accurate estimate of both its magnitude and distribution over the wing is still unavailable for dragonflies.

We assumed that average lift generated by a forewing during a flapcycle is equal to $1 / 4$ of the weight $W$ of the insect (because of its four wings) and equal during down- and upstroke:

$\bar{L}=\frac{1}{4} \cdot W$

The aerodynamic load model was based on blade element theory [40, 42]:

$\bar{L}=\frac{\rho_{\text {air }}}{2 T} \int_{0}^{T} \int_{0}^{r} \int_{0}^{c}(\dot{\varphi}(t) \cdot x)^{2} \cdot C_{l}(x) d y d x d t$

Here, $\mathrm{T}(=1 / \mathrm{f})$ is the period of one flapcycle, $\rho_{\text {air }}$ the air density at sea level $(1.225 \mathrm{~kg} / \mathrm{m} 3), x$ the spanwise distance from the root, $r$ the wing length, $y$ the position on the chord $c$, and $t$ is time. We scale the lift from a unit elliptical lift distribution, with maximal lift at the base and zero lift at the wing tip, which is typical for fixed wing aircraft, but most likely a substantial simplification for insect wings. The angular velocity of the wing is:

$$
\dot{\varphi}(t)=\omega_{\text {flap }} \cdot \Phi \cdot \cos \left(\omega_{\text {flap }} t\right)
$$

To distribute the lift over the wing we first distributed the lift spanwise over wing chord sections with width $\mathrm{d} x$ using the chord, speed and lift coefficient distribution. Next we distributed the spanwise lift distribution $C_{l}(x)$ chordwise using the following model for the chordwise pressure distribution:

$C_{l}(x)=\int_{0}^{c} C_{p}(x, y) d y$

The chordwise pressure coefficient distribution over the wing $C_{\mathrm{p}}(\mathrm{x}, \mathrm{y})$ is modeled based on two second-order polynomials. The first polynomial $\left(P_{1}(y)=\alpha 0+\alpha 1 \cdot y+\right.$ $\left.\alpha 2 \cdot y^{2}\right)$ is defined from the leading edge $\left(y_{L E}=0\right)$ to the aerodynamic centre $\left(y_{a c}\right)$ and the second polynomial $\left(P_{2}(y)=b 0+b 1 \cdot y+b 2 \cdot y^{2}\right)$ from $y_{a c}$ to the trailing edge $\left(y_{T E}=C\right)$. The peak of the polynomial distribution is assumed to be at $y_{a c}$ (at $1 / 4$ th of the chord length from the leading edge). The pressure difference distribution can now be calculated via:

$\Delta P(x, y)=\frac{\rho_{\text {air }}}{2}(\omega(t) \cdot x)^{2} \cdot C_{p}(x, y)$

In this way we obtain a double curved pressure distribution over the wing area, which depends on the local pressure coefficient, angular velocity, spanwise position and time. For our FEM analysis we determined the average pressure load for all individual shells. In this we assumed that the pressure distribution over the shell is uniform.

\section{Inertial load model}

Inertial loads are caused by accelerating wing mass, we considered accelerations due to stroke and neglected accelerations due to angle of attack changes. The inertial loads due to stroke are calculated according to:

$F(x, t)=\ddot{\varphi}(t) \cdot x \cdot\left(\frac{d m}{d x}\right) d x$

Here the angular acceleration is modeled based on a sinusoidal stroke:

$$
\ddot{\varphi}(t)=-\omega_{\text {flap }}^{2} \cdot \Phi \cdot \sin \left(\omega_{\text {flap }} t\right)
$$

For our FEM the local masses are calculated for each element: We multiplied the volumes of each beam and shell element with the cuticle density $\rho_{c}$. The accelerations due to wing stroke were calculated at the centre of 


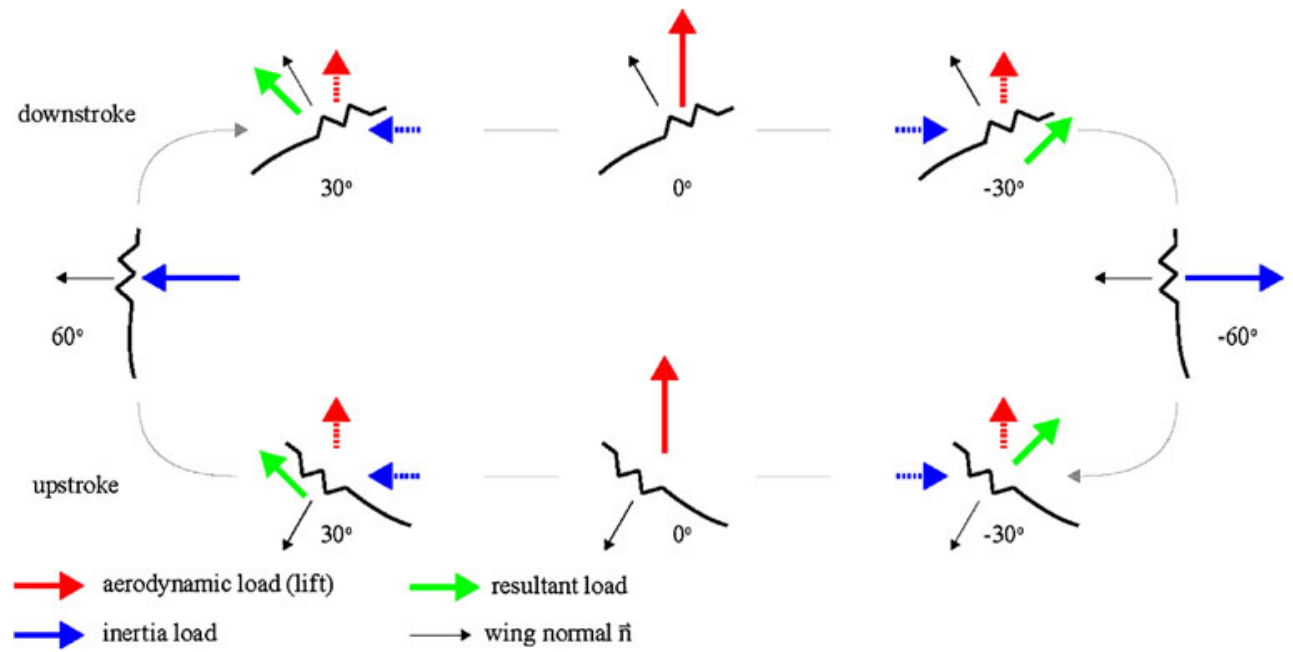

Fig. 6 Eight load cases during hovering flight. Inertial loads are in the horizontal direction and reverse during stroke reversal (pronation and supination). During stroke reversal the inertial loads act perpendicularly to the wing surface. The lift and inertial loads build up the resultant load vector. The indicated angles represent stroke positions

each element. We took the mass of haemolymph in hollow veins into account, thereby taking haemolymph density equal to cuticle density. We divided the inertial force by element area to obtain inertial 'pressure loads' on each element.

\section{Load cases}

We analyzed four load cases that occur during the upstroke and four that occur during during downstroke of the wing (Fig. 6). The difference between the four upstroke and downstroke load cases is the orientation of the airfoil with respect to the loading; this is significant, because an insect airfoil is asymmetric. The loadcases were defined as follows; (i) Maximum aerodynamic lift loading, which occurs at midstroke $\varphi=0^{\circ}$, in our model, this lift points upwards opposing gravity during the upstroke and downstroke. (ii) Maximum load due to inertia occurs in the horizontal plane at stroke reversal (at $\varphi= \pm 60^{\circ}$ ) during supination and pronation. (iii) At $\varphi= \pm 30^{\circ}$ four combined aerodynamic and inertial load cases were studied during both acceleration and deceleration of the wing for the upstroke and downstroke case. The resulting load directions depend on the combination of the aerodynamic and inertial load, while the orientation of the airfoil depends on the flapping phase; downstroke $v s$. upstroke.

\section{Results and Discussion}

Micro computed tomography scanning proved to be a successful method to digitise a dragonfly forewing in three dimensions. Subsequently created thickness distributions showed decreasing thickness of veins and membranes from root to tip and from leading to trailing edge. We analysed results from the numerical analysis using custom Matlab algorithms. Maximum inertial loads appeared higher than maximum aerodynamic loads in spanwise direction. Structural deformations were small, the maximum deflections were found during stroke reversal $(1.5 \mathrm{~mm})$ and were located at the wing tip. The numerical analyses also revealed that wing bending during upstroke was higher when compared to downstroke. The latter is likely caused by the asymmetrical shape of the wing due to camber and positions of the valleys and hills in the wing build up by radial veins [17]. Our vibration analysis shows that the wing's first natural

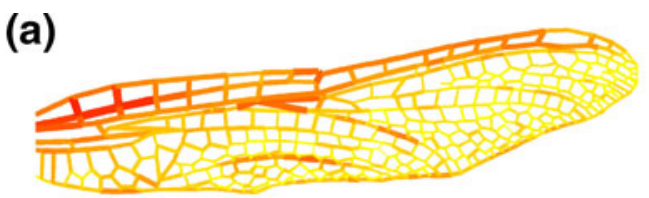

(b)

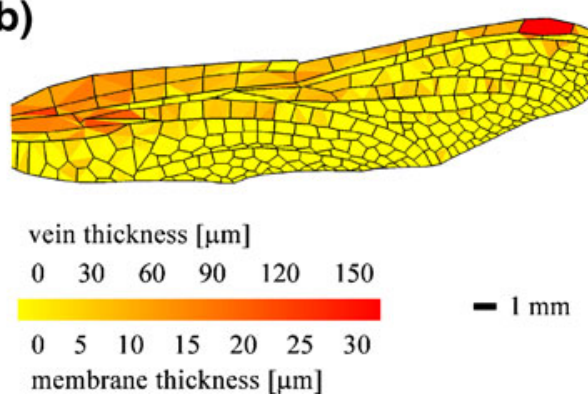

Fig. 7 Thickness distribution of the forewing of Sympetrum vulgatum. (a) Thickness distribution of the veins. (b) Thickness distribution of the membranes 


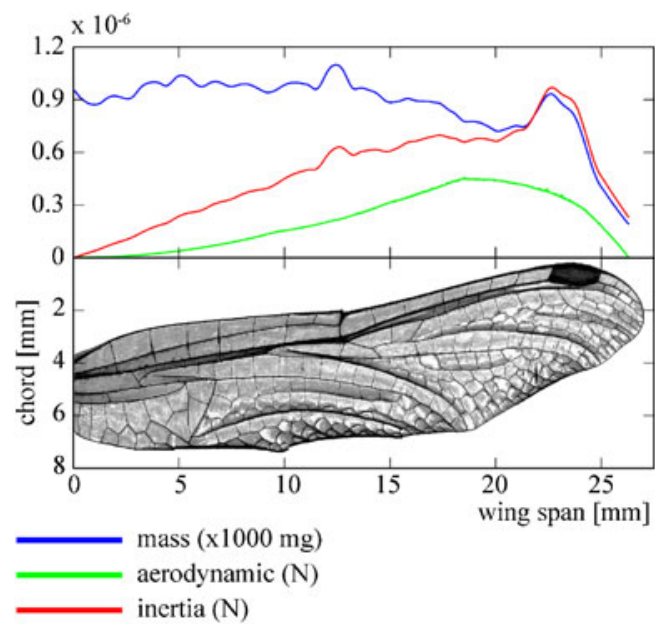

Fig. 8 Mass, and maximum aerodynamic and inertial load distribution along the single wing span. Local mass and inertial load maxima appear at the wing nodes and pterostigma

frequency in vacuum is approximately 4.8 times higher than the flapping frequency of Sympetrum vulgatum.

\section{Digitisation and Image Analysis of a Dragonfly Wing}

Our results show that three-dimensional digitisation of thick insect wings, such as those from dragonflies, is possible when using modern micro-CT scanners. Micro-CT proved to be a relatively easy, fast and efficient way to capture the morphology of a dragonfly wing (Figs. 3, 4). The only downside of micro CT-scanning is that our wings were slightly deformed near the trailing edge, because the wings needed to be dried to reduce scatter due to evaporation in the warm scanner. The deformations due to drying most likely affected wing stiffness to some degree. The trailing edge veins and membrane are, however, significantly thinner than the rest of the wing, which decreases the effect of local deformation on wing stiffness, because it balances their relative large distance with respect to the centroid of the wing. We determined the thickness distribution of the wing (Fig. 7) based on the crosssectional image stack of the wing. As expected, thick veins are located near the wing root and the leading edge. Some of the continuous veins that span from leading to trailing edge are thicker as well. The thicknesses of thick veins range from 75 to $150 \mu \mathrm{m}$. The veins that form the hexagonal pattern are thinner, with thickness ranging from 10 to $50 \mu \mathrm{m}$. The thickness distribution of membranes is similar; thick membranes near the leading edge and root, ranging between 15 and $25 \mu \mathrm{m}$. Thin membranes range in thickness from $3.6 \mu \mathrm{m}$ up to $15 \mu \mathrm{m}$. The hollow pterostigma of the wing has a maximum thickness, measured from the bottom to top side of the pterostigma, of approximately $220 \mu \mathrm{m}$.
Fig. 9 Analytically modelled spatial aerodynamic pressure and inertial load distribution over the forewing area during a stroke in hovering flight. Aerodynamic load shows a spatial peak at $80 \%$ of the wing length. Due to maximal translational motion at midstroke $\left(\varphi=0^{\circ}\right)$, the aerodynamic load is maximal whereas inertial loads are zero. At stroke reversal, proand supination, the inertial load is maximal and the aerodynamic load is zero. Vein inertial loads dominate the total inertial load

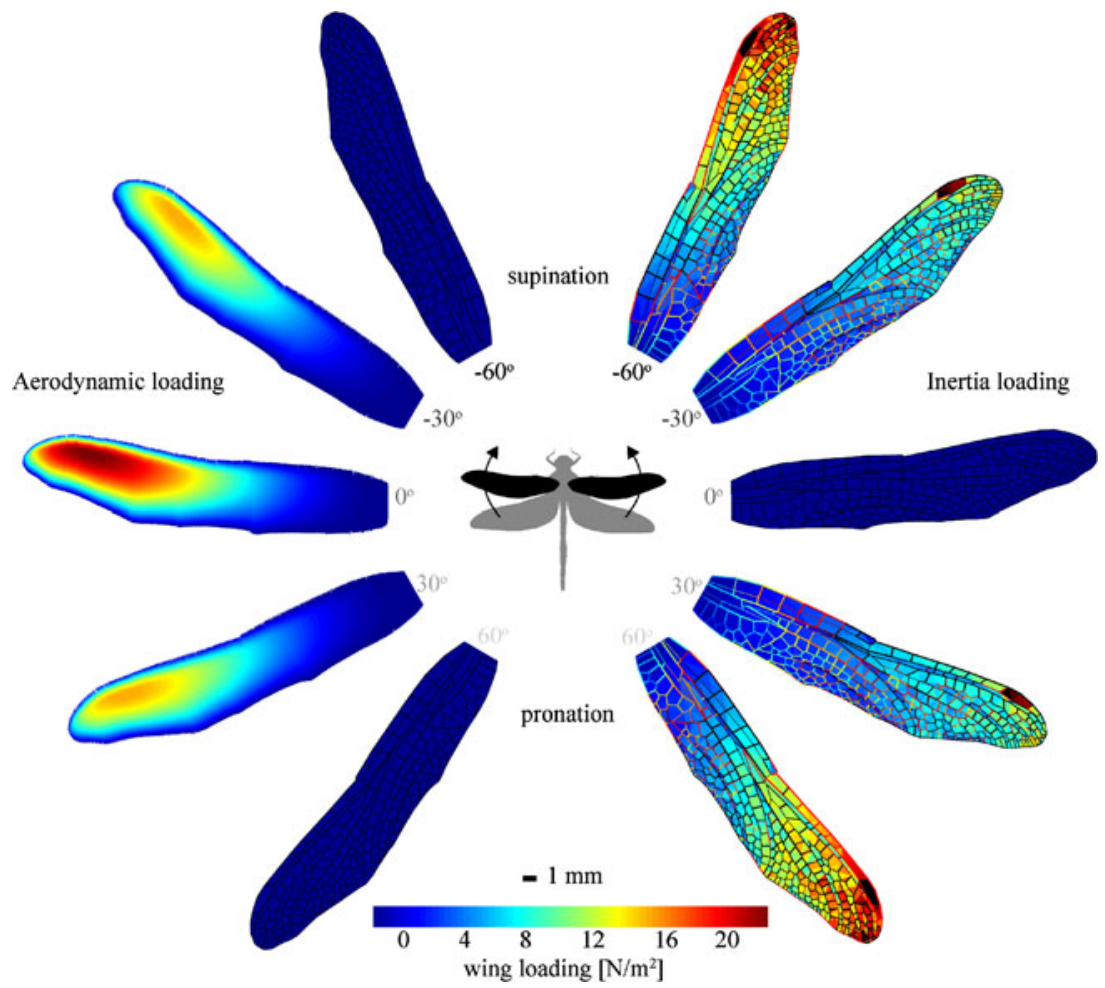




\section{Load Distributions}

We compared the maximum aerodynamic load at midstroke with the maximum inertial load at stroke reversal. The loads are summed along the chord to obtain a spanwise distribution along the wing (Fig. 8). The distributions are similarly shaped from root to tip, with local maxima for inertial load at the nodus $( \pm 45 \%$ wing length $r$ ) and pterostigma $( \pm 85 \% r)$. The maximum aerodynamic load is found at about $65 \%$ of the wingspan $(r)$. We found that the inertial forces along the wingspan are approximately 1.5-3 times higher than the aerodynamic forces. This matches the estimation of Ennos [31] that spanwise bending moments due to the inertia of flapping wings are about twice as large as those due to aerodynamic forces. The simultaneously plotted mass distribution shows that local spanwise mass maxima appear at the wing nodus and the pterostigma. The peaks suggest that these areas have a relatively large influence on inertial deformation responses.

The response of the wing structure to the spatial distribution of aerodynamic and inertial loads throughout half a flapcycle are shown in Fig. 9. The wing's angular and translational velocity is zero during stroke reversal, aerodynamic loads are therefore not present in our model. At midstroke the wing's acceleration is zero, thus inertial loads are zero, while aerodynamic forces peak at midstroke. The peak of the aerodynamic pressure distribution is located at

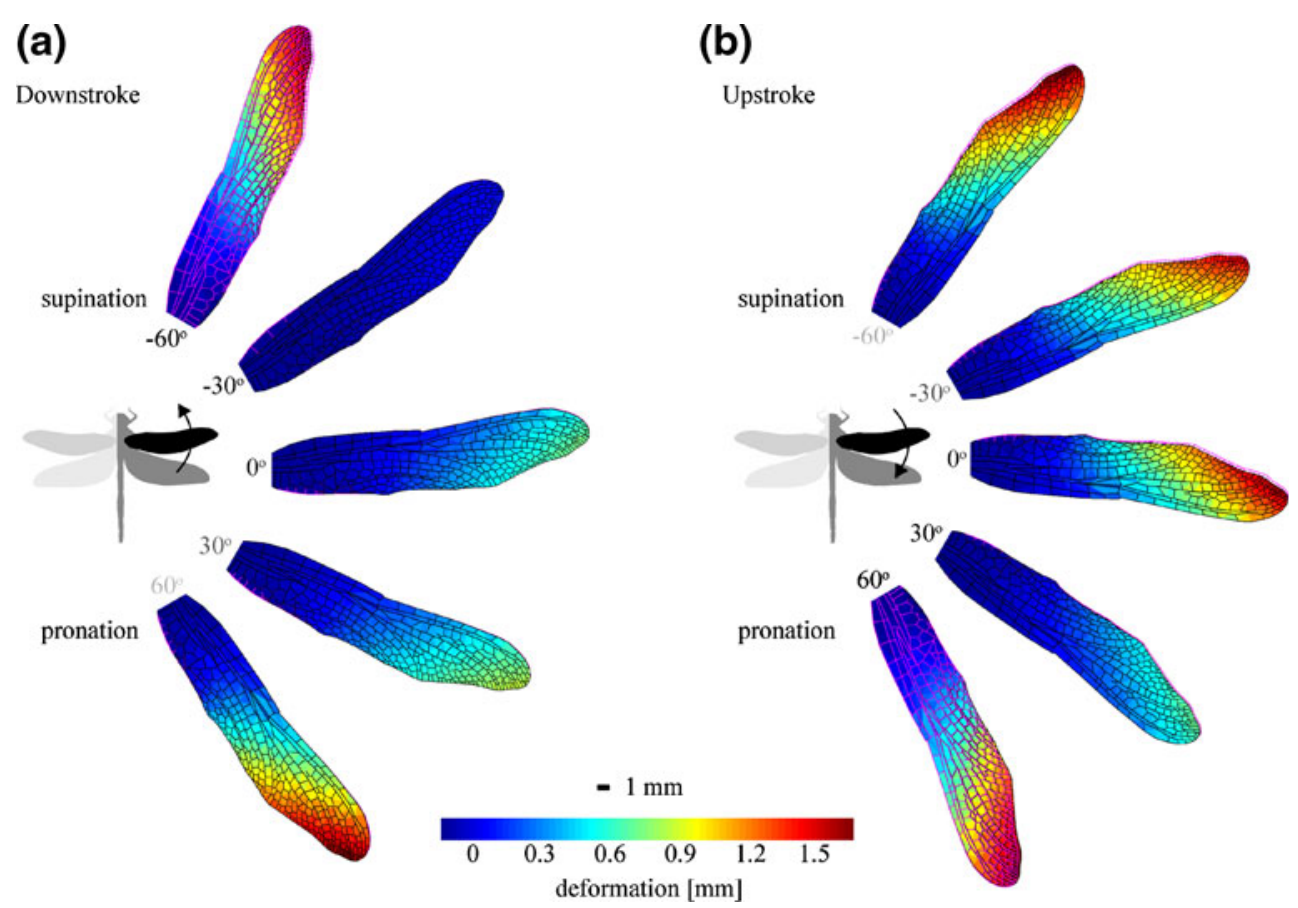

Fig. 10 Spatial deformations (shown 10 times enlarged) of a flapping Sympetrum vulgatum wing during hovering flight. Magenta plots show undeformed shape. (a) Deformations during downstroke. (b) Deformations during upstroke. Deformation plots during pro- and supination are similar in (a) and (b) but plotted from another point of view. Deformations during upstroke are approximately twice as large compared to the downstroke. The largest deformation is found during stroke reversal; $1.5 \mathrm{~mm}$. Before stroke reversal during down- and upstroke only small deformations are found because loads are applied almost in plane of the wing (Fig. 6)

$\pm 80 \%$ of the wing length. The spatial inertia distribution shows that inertial loads caused by membrane elements gradually rise to the wing tip where they reach maximum at the pterostigma $( \pm 85 \% r)$. The mass of veins causes high inertial forces from root to the tip, especially during stroke reversal when inertial loading is maximal.

\section{Wing Deformation}

We analyzed the wing deformation under aerodynamic and inertial loading throughout the stroke cycle for the eight loadcases shown in Fig. 6. Figure 10 shows that the wing deformation during the translational phase of the upstroke is generally 2 times higher than the deformation during downstroke. This suggests that the asymmetric wing architecture responds more stiffly against loads occurring during downstroke than during upstroke. This observation corresponds to findings of Wootton et al. [16] who show that cambered wings exhibit asymmetric bending deformations; the wing being more rigid to forces applied from the concave side than from the convex side. At supination and pronation the wing also deflects approximately 2 times more than at the midpoint of the downstroke. The difference in deformation is primarily due to the inertial loading at stroke reversal being higher than the aerodynamic loading at midstroke. Relatively small deformations are found before stroke reversal (at $\varphi=-30^{\circ}$ during

\section{(b)}


downstroke and $\varphi=30^{\circ}$ during upstroke). This is probably due to the direction of the resultant load on the wing (Fig. 6). The resultants are in these cases directed $\pm 15^{\circ}$ in the plane of the wing and therefore hardly cause deformation, because the wing's second moment of inertia is very high in this direction. The overall deformations caused by the applied loads are generally relatively small compared to the wing length. The maximum lateral displacement found is $1.5 \mathrm{~mm}$ which is $5.5 \%$ of the wing length and occurs during supination. Overall bending patterns are remarkably similar. Both aerodynamic and inertial load distributions induce twist towards the wingtip and increase bending of the trailing edge. The maximum twist found during supination is approximately $7^{\circ}$, measured at $75 \%$ of the wing length. This twist due to wing lift reduces the effective aerodynamic angle of attack of the wing, which will reduce lift and help stabilize wing deformation under aerodynamic loading.

\section{Wing Vibration}

Results of free vibration analyses, in which we neglect damping, show the same deformation for the natural modes as Wootton et al. [16] found; bending for the first natural mode and torsion for the second natural mode (Fig. 11). In our three-dimensional model these natural vibration modes occur at frequencies 4.8 (bending) and 9.3 (torsion) times higher than the insect's flapping frequency $f_{\text {flap }}(32.3 \mathrm{~Hz})$, see Table 1 . In our two dimensional model (z-dimensions flattened) the first natural frequency $f_{n}(=25.4 \mathrm{~Hz})$ is close to the flapping

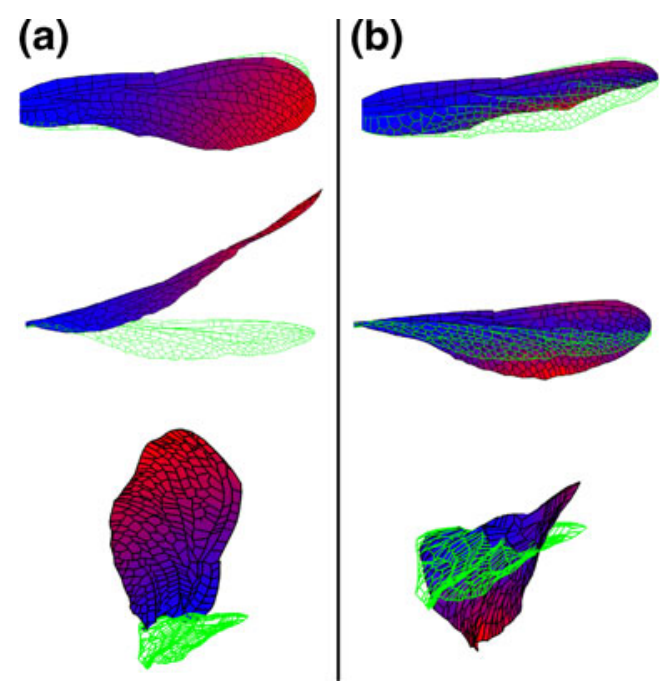

Fig. 11 First and second natural vibration modes of the dragonfly forewing in vacuum (top, hind and side view). (a) The first natural vibration mode of Sympetrum vulgatum. Deformation is dominated by bending. (b) The second natural vibration mode of the wing. The deformation shows mainly torsion. Green depicts the wing's original shape
Table 1 Natural frequencies 3D and 2D forewing

\begin{tabular}{lccccl}
\hline $\begin{array}{l}\text { Wing } \\
\text { model }\end{array}$ & Mode $(\mathrm{n})$ & $f_{n}(\mathrm{~Hz})$ & $\omega_{\mathrm{n}}(\mathrm{rad} / \mathrm{s})$ & $\omega_{\mathrm{n}} / \omega_{\text {flap }}$ & Type \\
\hline 3D & 1 & 154 & 968 & 4.8 & Bending \\
& 2 & 301 & 1893 & 9.3 & Torsion \\
2D & 1 & 25.4 & 158 & 0.8 & Bending \\
& 2 & 118 & 739 & 3.7 & Torsion \\
\hline
\end{tabular}

frequency $f_{\text {flap }}(32.3 \mathrm{~Hz})$ (Table 1), this result is similar to the one obtained by Wootton et al. [16].

In our two-dimensional model the deformation responses are again bending for the first mode and torsion for the second mode. Our comparison of natural vibration modes of a realistic three dimensional wing architecture and a simplified two dimensional "flat" architecture shows that the three dimensionality is critical for determining if insects flap their wings at frequencies lower, equal or higher than the resonant frequency of the wing. Our results show that Sympetrum vulgatum is flapping its forewing at a frequency roughly 5 times lower than the first bending mode and 9 times lower than first torsion mode of its forewing.

The structural and aerodynamic damping that a real wing in air will experience will lower the natural vibration frequencies we find to some degree. We estimate the reduction using the following standard equation for damped vibrating systems:

$\omega_{d}=\omega_{n} \cdot \sqrt{1-\zeta^{2}}$

Here, $\omega_{d}$ is the damped natural frequency, $\omega_{n}$ the undamped natural frequency and $\zeta$ the damping ratio. We calculate that $\zeta$ needs to be as high as 0.98 to lower the lowest natural frequency enough to make it identical to the flapping frequency. Experiments carried out by Combes and Daniel [15] with insect wings vibrating in air and helium show that damping has a minimal effect on the vibration modes of insect wings. They find that damping is only slight and primarily due to structural damping caused by material hysteresis and damping due to the fluid in the wings veins, supporting the vacuum approximation. Based on these experiments we conclude that damping coefficients of 0.98 are highly unrealistic; realistic damping ratios will be much closer to zero. Therefore we expect that our forewing vibration analysis will be realistic for dragonflies in hovering flight.

\section{Conclusions}

We found that micro-CT scanning is a very promising method for measuring and digitizing the wings, veins and 
membranes of insects. Whereas current state of the art micro-CT scanners are limited to large insects wings, we foresee that any insect wing could be successfully scanned in the near future as the resolution of micro-CT scanners increases every year. The main challenge for accurately digitizing the wings $3 \mathrm{D}$ architecture is minimizing the deformation due to drying of the wing needed to reduce scatter and noise in the scan due to evaporation. Our scan of a dragonfly Sympetrum vulgatum forewing shows that vein and membrane thickness increases from tip to root, which allows the wing to effectively bear both inertial and aerodynamic loads. Inertial loads along the wingspan are approximately 1.5-3 times higher than the aerodynamic loads. As a result the wings deformation is dominated by inertial loading, which is maximal during stroke reversal. Comparing wing deformation during up- versus downstroke we find that the wings asymmetry due to camber causes the wing being more rigid to forces applied from the concave side than from the convex side. We find that wing bending is low during hovering as the maximum lateral displacement is $5.5 \%$ wing span. Finally we find that dragonflies flap their forewing at frequencies significantly lower than the first natural vibration mode of the forewing, 5 times lower for Sympetrum vulgatum. The strategy of dragonflies to flap their wings at a much lower frequency than the natural vibration frequency of their forewing is contrasted by the most advanced flapping wings designed for micro air vehicles ([1-3]; e.g. see Fig. 1). Of these micro air vehicles the smallest ones operate at the scale of dragonflies [2]. All these flapping wing designs operate at much higher frequencies than the natural frequencies of their wing, which are extremely low because of the slack, sail-like, wingdesign. These slack wings are easy to actuate back and forth and simply deform aeroelastically [3] such that the geometric angle of attack of the wing is of the right order of magnitude to generate both lift and thrust. A much stiffer wing, like the one of dragonflies, requires a more sophisticated aeroelastic design and actuation of the wings. Stiffer and more precisely designed wings could, however, yield higher performance because less energy might be dissipated due to suboptimal wing deformation and angle of attack that result from the simple sail-like wings of many flapping MAVs. In contrast, the wing deformation and angle of attack of the much stiffer dragonfly-like wings are under more precise control through high fidelity aeroelastic tailoring.

Acknowledgements The authors would like to thank M. Muller for his enthusiastic support and help with capturing and identifying dragonfly specimens and E. van de Casteele at SkyScan for scanning the dragonfly wings. We thank A. Beukers, J.L. van Leeuwen, V. Antonelli, S. Koussios for support and valuable comments. We thank J.F.V. Vincent and S.A. Combes for critically reading our manuscript. We acknowledge F.G.C. Oostrum, for producing the SEM images, H. Schipper for help with sectioning, A. Kooijman for help with exploring 3D laser scanning techniques. Finally we thank three anonymous reviewers for their helpful comments and suggestions.

Open Access This article is distributed under the terms of the Creative Commons Attribution Noncommercial License which permits any noncommercial use, distribution, and reproduction in any medium, provided the original author(s) and source are credited.

\section{References}

1. Pornsin-Sirirak TN, Tai YC, Ho CH and Keennon M (2001) Microbat-a palm-sized electrically powered omithopter. NASA/ JPL Workshop on Biomorphic Robotics, Pasadena, USA.

2. Kawamura Y, Souda S, Nishimoto S and Ellington CP (2008) Clapping-wing micro air vehicle of insect size. In: Kato $\mathrm{N}$ and Kamimura S (eds) Bio-mechanisms of swimming and flying. Springer Verlag, pp 319-330

3. Lentink D, Jongerius SR and Bradshaw NL (2009) The scalable design of flapping micro air vehicles inspired by insect flight In: Floreano D, Zufferey JC, Srinivasan MV and Ellington CP (eds) Flying Insects and Robots. Springer-Verlag

4. Zhao L, Huang Q, Deng X, Sane S (2009) Aerodynamic effects of flexibility in flapping wings. J R Soc Interface 7:485-497

5. Rees CJC (1975) Aerodynamic properties of an insect wing section and a smooth aerofoil compared. Nature 258:141-142

6. Rees CJC (1975) Form and function in corrugated insect wings. Nature 256:200-203

7. Okamoto M, Yasuda K, Azuma A (1996) Aerodynamic characteristics of the wings and body of a dragonfly. J Exp Biol 199:281-294

8. Kesel AB (2000) Aerodynamic characteristics of dragonfly wing sections compared with technical aerofoils. J Exp Biol 203:3125-3135

9. Tamai M, Wang Z, Rajagopalan G, Hu H and He G (2007) Aerodynamic performance of a corrugated dragonfly airfoil compared with smooth airfoils at low Reynolds numbers. 45th AIAA Aerospace Sciences Meeting and Exhibit. Reno, Nevada, 1-12.

10. Smith MJC (1996) Simulating moth wing aerodynamics: towards the development of flapping-wing technology. AIAA Journal 34:1348-1355

11. Kesel AB, Philippi U, Nachtigall W (1998) Biomechanical aspects of the insect wing: an analysis using the finite element method. Comput Biol Med 28:423-437

12. Herbert RC, Young PG, Smith CW, Wootton RJ, Evans KE (2000) The hind wing of the desert locust (Schistocerca gregaria Forskål). III. A finite element analysis of a deployable structure. J Exp Biol 203:2945-2955

13. Combes SA, Daniel TL (2003) Flexural stiffness in insect wings. I. Scaling and the influence of wing venation. J Exp Biol 206:2979-2987

14. Combes SA, Daniel TL (2003) Flexural stiffness in insect wings. II. Spatial distribution and dynamic wing bending. J Exp Biol 206:2989-2997

15. Combes SA, Daniel TL (2003) Into thin air: contributions of aerodynamic and inertial-elastic forces to wing bending in the hawkmoth Manduca sexta. J Exp Biol 206:2999-3006

16. Wootton RJ, Herbert RC, Young PG, Evans KE (2003) Approaches to the structural modelling of insect wings. Phil Trans R Soc B 358:1577-1587

17. Wootton RJ (1995) Geometry and mechanics of insect hindwing fans: a modelling approach. Biological Sciences, Proceedings, pp 181-187

18. Ellington CP (1999) The novel aerodynamics of insect flight: applications to micro-air vehicles. J Exp Biol 202:3439-3448 
19. Wootton and Kukalova-Peck (2000) Flight adaptations in Palaeozoic Palaeoptera. Biol Rev 75:129-167

20. Rowe RJ (2004) Dragonfly flight. http://tolweb.org/notes/? note_id=2471. Accessed 10 April 2007.

21. Tsuyuki K, Sudo S, Tani J (2006) Morphology of insect wings and airflow produced by flapping insects. J Intell Mater Syst Struct 17:743-751

22. Lanczos C (1956) Applied Analysis. Prentice-Hall, Englewood Cliffs, NJ

23. Wainwright SA, Biggs WD, Currey JD and Gosline JM (1982) Mechanical design in organisms. Princeton University Press.

24. Vincent JFV, Wegst UGK (2004) Design and mechanical properties of insect cuticle. Arthropod Stru Dev 33:187-199

25. Smith CW, Herbert R, Wootton RJ, Evans KE (2000) The hind wing of the desert locust (Schistocerca gregaria Forskål). II. Mechanical properties and functioning of the membrane. J Exp Biol 203:2933-2943

26. Gorb SN (1999) Serial elastic elements in the damselfly wing: mobile vein joints contain resilin. Naturwissenschaften 86:552-555

27. Haas F, Gorb SN, Blickhan R (2000) The function of resilin in beetle wings. Proc R Soc Lond B 267:1375-1381

28. Rüppell G (1989) Kinematic analysis of symmetrical flight manoeuvres of Odonata. J Exp Biol 144:13-42

29. Ellington CP (1984) The aerodynamics of hovering insect flight. II. Morphological parameters. Phil Trans R Soc Lond B 305:17-40

30. Wakeling JM, Ellington CP (1996) Dragonfly flight II. Velocities, accelerations and kinematics of flapping flight. J Exp Biol 200:557-582

31. Ennos R (1988) The inertial cause of wing rotation in Diptera. J Exp Biol 140:161-169
32. Sane SP, Dickinson MH (2001) The control of flight force by a flapping wing: lift and drag production. J Exp Biol 204:26072626

33. Lehmann FO, Dickinson MH (1998) The control of wing kinematics and flight forces in fruit flies (Drosophila spp.). J Exp Biol 201:385-401

34. Daniel TL, Combes SA (2002) Wings and fins: bending by inertial or fluid-dynamic forces? Integr Comp Biol 42:1044-1049

35. Ellington CP, van den Berg C, Willmott AP, Thomas ALR (1996) Leading-edge vortices in insect flight. Nature 384:626-630

36. Van den Berg C, Ellington CP (1997) The vortex wake of a 'hovering' model hawkmoth. Phil Trans R Soc Lond B 352:317328

37. Van den Berg C, Ellington CP (1997) The three-dimensional leading-edge vortex of a 'hovering' model Hawkmoth. Phil Trans R Soc Lond B 352:329-340

38. Dickinson MH, Lehmann FO, Sane SP (1999) Wing rotation and the aerodynamic basis of insect flight. Science 284:1954-1960

39. Lehmann FO (2004) The mechanisms of lift enhancement in insect flight. Naturwissenschaften 91:101-122

40. Ellington CP (1984) The aerodynamics of hovering insect flight. I. The quasi-steady analysis. Phil Trans R Soc Lond B 305:1-15

41. Ellington CP (1984) The Aerodynamics of Hovering Insect Flight. III. Kinematics. Phil Trans R Soc Lond B 305:41-78

42. Osborne MFM (1951) Aerodynamics of flapping flight with application to insects. J Exp Biol 28:221-245

43. Bechert DW, Meyer R, and Hage W (2000) Drag reduction of airfoils with miniflaps. Can we learn from dragonflies? AIAA 130. 